\title{
Spinal cord injury and COVID-19: some thoughts after the first wave
}

\author{
Judith Sánchez-Raya ${ }^{1}$ Júlia Sampol $\mathbb{B}^{2}$
}

Received: 7 July 2020 / Accepted: 8 July 2020 / Published online: 21 July 2020

(c) International Spinal Cord Society 2020

The global outbreak of coronavirus SARS-CoV-2 disease (COVID-19) in December 2019 has created an unprecedented challenge to the world and especially to the health system. All levels of medical attention are being affected: from emergency departments, intensive care units, and medical wards to postacute, outpatient, primary and home care settings. Some years ago, the World Health Organization established as a priority the need to help health providers and governments to ensure that people with disability such as spinal cord injury (SCI) could always access the healthcare services in emergency situations [1]. However, the current pandemic constitutes a major challenge that has forced the development of new specific recommendations [2]. In people with the impact of COVID-19 has not yet been completely evaluated but, in an unprecedented effort, the medical community has developed many proposals aimed at improving the care of this group and their caregivers [3].

Still with a very recent experience, some reflections are emerging that we believe could apply to other SCI units. The main concerns are: (1) What is the real impact of COVID-19 in people with SCI and their caregivers? and (2) What measures or changes in the circuits of care and in our structures will be necessary in the future?

Problems derived from the reduced access to healthcare services and the reorganization of hospitals have been the norm for the past few months. Our department, provided with 20 beds in usual conditions, is the reference unit for SCI in Catalonia. It belongs to a tertiary hospital that, with

Júlia Sampol

jusampol@gmail.com

1 Physical Medicine and Rehabilitation Department, Hospital Universitari Vall d'Hebron, Barcelona, Spain

2 Pneumology Department, Hospital Universitari Vall d'Hebron, Barcelona, Spain
2300 COVID-19 admitted patients, has been one of those that has attended to a greater number of cases in Spain. This forced the hospital authorities to restructure the allocation of beds, increasing the number of critical care beds by five times. A similar reorganization has been reported in published works from Italy [4], Spain [5], or the USA [6]. In our unit, the number of beds was reduced to $50 \%$, specific rehabilitation areas were closed and early hospital discharge and the use of remote systems in outpatient care were encouraged. The adopted contingency plan had urgent care for acute SCI and for serious complications in patients with chronic SCI as its main goal.

Fortunately, the lockdown decreed on 14 March, 2020 in Spain, with a duration of almost 90 days, has led to a notable reduction in urgent consultation for SCI, as has been observed in other pathologies such as stroke [7] or ischemic heart disease [8]. During this period, the number of admissions for acute or chronic SCI has also decreased to a third of the usual rate of admissions in our unit. This phenomenon could be attributed to the reduced mobility of the population and consequently the risk of accidental trauma in the case of acute SCI and to a reluctance to consult in the case of people with chronic SCI as well.

Among those admitted with acute SCI, only one patient upon admission had COVID-19. Interestingly, in this patient the infection was asymptomatic and was only detected through the polymerase chain reaction (PCR) screening protocol of all admitted patients established in our center since the start of the outbreak. This fact highlights the need for these screening protocols to reduce the possibility of in-hospital outbreaks. However, at the beginning of the pandemic, we had four patients who contracted nosocomial SARS-CoV-2 infection: one patient who suffered a trauma at home with an acute SCI and three patients with chronic SCI. The four patients were infected despite in-hospital control measures of COVID-19 which included, in addition to screening at admission, the preservation of non-COVID areas and the use of adequate personal protection equipment 
by healthcare workers. During the same period, a nurse and three doctors from our unit were infected with SARS-COV2. This highlights the difficulty of avoiding infections in the current pandemic and the need to develop new strict isolation protocols for people with SCI, which should probably include structural changes in the units with the availability of single rooms with adequate ventilation systems.

Respiratory involvement is the main complication of severe COVID-19 cases. People with SCI have an increased risk of developing respiratory complications $[9,10]$ and frequently present comorbidities such as hypertension and obesity that have been linked to a worse prognosis of COVID-19 [11]. In our unit, four patients with SCI and nosocomial COVID-19 presented with severe disease: two were over 80 years old had centromedullar lesions and relevant comorbidity and both unfortunately died. However, the remaining two have survived to date: a 57-year-old male patient with no pathological history with a C2 AIS D cervical injury, severe oropharyngeal dysphagia who required enteral nutrition with a nasogastric tube and a 41-year-old male patient with acute traumatic SCI without comorbidities and a T5 AIS B neurological level who developed acute respiratory failure secondary to pneumonia and presented a favorable evolution with the appropriate treatment that included support measures with administration of high concentrations of oxygen and assisted coughing techniques. These results suggest that advanced age and comorbidity are risk factors for a poor prognosis in COVID-19 patients with SCI in a similar way to the general population [12]. Nevertheless, some of these patients may have a favorable evolution with adequate care and appropriate ventilatory support.

In admitted patients with SCI, special attention should be paid to any change in the baseline status, such as increased spasticity, the onset of an unwarranted low-grade fever or an increase in dysreflexia crisis or baseline neuropathic pain, in which case the diagnosis of a possible SARS-CoV2 infection should be evaluated by PCR. Preventive and vigilance measures for pneumonia, which frequently presents without typical symptoms in people with SCI, should be implemented, especially in cervical lesions. Specific respiratory muscle training and the application of mechanical assisted cough techniques are also important to reduce the chances of infection. In addition, the frequent use of noninvasive or invasive ventilation systems with passive leakage systems in patients with SCI require the adoption of appropriate technical modifications to avoid nosocomial outbreaks of the disease [13].

In relation to the lockdown measures and the fear of going to the hospital, people with chronic SCI have been less inclined to attend outpatient appointments. This fact involves the danger of worsening complications that usually occur in these patients. Both during the peak of the epidemic as well as in the current phase of lower viral transmission, the need to establish a remote follow-up of these patients arises. In our case, the medical team has proactively contacted patients by telephone, followed up on the results of tests and cultures and attended to queries mainly regarding respiratory complications, dysreflexia, neurogenic intestine, and pressure ulcers. Many of them have been effectively treated and, as a potentially positive consequence of the current crisis, telemedicine could be an essential tool to provide care and services to people with SCI in the future thereby avoiding the inconvenience and risks of traveling to the hospital [14]. This should be accompanied by better patient and caregivers' education and protocolization of follow-up guidelines. This new approach could lead to a greater empowerment of people with SCI and their caregivers and a better control of common problems thus avoiding the need for urgent care and/or hospital admissions [15]. Additional strategies should include specific measures to help people with SCI and their caregivers mantain adequate hand washing and hygiene minimizing the risk of infection spread from carers and the adequate disinfection of the utensils they need in their daily lives such as wheelchairs. Furthermore, an improved organization of caregivers, probably involving an increased number of people, in case caregivers develop COVID-19 symptoms and need to isolate themselves.

Our previous reflections have arisen from the point of view of an SCI unit integrated into a tertiary university hospital of a European public national system. However, we must remember that SCI is a global problem. Many people with SCI live in low- and middle-income countries [16], and medical care shows marked disparities. Most of our proposals are not feasible for many units and thus COVID-19 can be an aggravating factor of these differences. There is currently a strong uncertainty about the future of the pandemic, and rapid access to a potential vaccine by all people at high risk including those with SCI should be a priority. While we do not have it, the problems we are experiencing should inspire clinicians, researchers, and policy makers to redouble efforts to develop affordable strategies in the care of people with SCI.

In conclusion, the COVID-19 pandemic has been a major challenge for people with SCI and for the specialized units that care for them. The rapid transmission of the infection in most countries has forced a rapid reorganization of units like ours that are integrated into general hospital complexes. While the global emergency nature of the outbreak has forced restrictions in various aspects of medical care, it is also true that some of the solutions found in this period could be consolidated and contribute to a positive evolution of the healthcare organization. It should not be forgotten that the COVID-19 pandemic is affecting the five continents and countries with a very diverse level of development and 
health system organization. Therefore, the prevention of infection and its treatment must be adapted to the different situations in which people with SCI live. In places where specialized units are not available, the recommendation would be to take extreme protection measures to avoid contagion, to use noninvasive respiratory support measures and, when possible, to grant access to intensive care units. In the immediate future, it will be very important to publish the studies carried out during de COVID-19 pandemic and to share the lived experiences in the specialized debate forums in order to broaden our knowledge and prepare our units for eventual future emergencies.

\section{Compliance with ethical standards}

Conflict of interest The authors declare that they have no conflict of interest.

Publisher's note Springer Nature remains neutral with regard to jurisdictional claims in published maps and institutional affiliations.

\section{References}

1. World Health Organization. Guidance note on disability and emergency risk management for Health. Geneva, World Health Organization; 2013. https://www.who.int/hac/techguidance/prepa redness/disability/en/.

2. World Health Organization. Disability considerations during the COVID-19 outbreak. Geneva, World Health Organization; 2020. https://www.who.int/publications/i/item/WHO-2019-nCoV-Disa bility-2020-1.

3. The International Spinal Cord Society. COVID-19 resources for ISCoS members and spinal cord injury professionals. Aylesbury, The International Spinal Cord Society. https://www.iscos.org.uk/ covid-19-resources-.

4. Boldrini P, Bernetti A, Fiore P. Impact of COVID-19 outbreak on rehabilitation services and physical and rehabilitation medicine (PRM) physicians' activities in italy. an official document of the Italian PRM society (SIMFER). Eur J Phys Rehabil Med. 2020. https://doi.org/10.23736/S1973-9087.20.06256-5.

5. López-Dolado E, Gil-Agudo A. Lessons learned from the coronavirus disease 2019 (Covid-19) outbreak in a monographic center for spinal cord injury. Spinal Cord. 2020;58:517-9. https:// doi.org/10.1038/s41393-020-0473-z.

6. Escalon M, Herrera J. Adapting to the COVID-19 pandemic in New York City. Am J Phys Med Rehabil. 2020;99:453-8. https:// doi.org/10.1097/PHM.0000000000001451.

7. Baracchini C, Pieroni A, Viaro F, Cianci V, Cattelan AM, Tiberio I, et al. Acute stroke management pathway during Coronavirus-19 pandemic. Neurol Sci. 2020;41:1003-5. https://doi.org/10.1007/ s10072-020-04375-9.

8. Huet F, Prieur C, Schurtz G, Gerbaud E, Manzo-Silberman S, Vanzetto $\mathrm{G}$, et al. One train may hide another: acute cardiovascular diseases could be neglected because of the COVID-19 pandemic. Arch Cardiovasc Dis. 2020;113:303-7. https://doi.org/ 10.1016/j.acvd.2020.04.002.

9. Sampol J, González-Viejo MÁ, Gómez A, Martí S, Pallero M, Rodríguez E, et al. Predictors of respiratory complications in patients with C5-T5 spinal cord injuries. Spinal Cord. 2020;1-6. https://doi.org/10.1038/s41393-020-0506-7.

10. Brown R, DiMarco AF, Hoit JD, Garshick E. Respiratory dysfunction and management in spinal cord injury. Respir Care. 2006;51:853-68.

11. Richardson S, Hirsch JS, Narasimhan M, Crawford JM, McGinn T, Davidson KW, et al. Presenting characteristics, comorbidities, and outcomes among 5700 patients hospitalized with COVID-19 in the New York city area. JAMA. 2020;323:2052-9. https://doi. org/10.1001/jama.2020.6775.

12. Zhou F, Yu T, Du R, Fan G, Liu Y, Liu Z, et al. Clinical course and risk factors for mortality of adult inpatients with COVID-19 in Wuhan, China: a retrospective cohort study. Lancet. 2020;395:1054-62. https://doi.org/10.1016/S0140-6736(20)30566-3.

13. National Health Service. Guidance for the role and use of noninvasive respiratory support in adult patients with coronavirus (confirmed or suspected). England, NHS 2020. https://www.engla nd.nhs.uk/coronavirus/wpcontent/uploads/sites/52/2020/03/specia lty-guide-NIV-respiratory-support-and-coronavirus-v3.pdf.

14. Watanabe TK, Esquenazi A, Flanagan S. The transformation of the rehabilitation paradigm across the continuum of care. PM R. 2018;10 (9 Suppl 2):S264-71. https://doi.org/10.1016/j.pmrj. 2018.08.381.

15. O'Connell CM, Eriks-Hoogland I, Middleton JW. Now, more than ever, our community is needed: spinal cord injury care during a global pandemic. Spinal Cord Ser Cases. 2020;6:18. https://doi. org/10.1038/s41394-020-0270-0.

16. GBD 2016 Traumatic Brain Injury and Spinal Cord Injury Collaborators. Global, regional, and national burden of traumatic brain injury and spinal cord injury, 1990-2016: a systematic analysis for the Global Burden of Disease Study 2016. Lancet Neurol. 2019;18:56-87. https://doi.org/10.1016/S1474-4422(18)30415-0. 